\title{
Measurements Standards and Test Signals in QRS Boundary Determination
}

\author{
S Hargittai
}

\author{
Innomed Medical Inc, Budapest, Hungary
}

\begin{abstract}
The most important step of quantitative ECG analysis is to determine the wave boundaries. The main objectives of this paper are to consider the scope and the limitations of using CSE databases and standard test signals for evaluation and improvement of ECG wave recognition programs. Three databases were used for evaluating the ECG measurement programs. The databases were not only used for automatic assessment but all records and channels were checked individually and deviations from the references were analyzed. The CSE databases and the standard analytical ECG curves can be used for automatic evaluation of an ECG measurement program, but reference data should be used with care. Design engineers have to have appropriate knowledge in both the signal-processing field and electrocardiography.
\end{abstract}

\section{Introduction}

The most important step of quantitative ECG analysis is the determination the primary fiducial points (onset and offset of QRS complex, P and T waves) of a heart cycle. Do we have exact and useful definitions of complexes, waves and their boundaries? We do not. Definition of wave by CSE Working Party - a wave is discernible deviation from the baseline, but for example the QRS-T baseline is determined in an interval before QRS onset [1]. Therefore for the detection of a wave we have to know the baseline, consequently the onset of the not yet detected wave. It is unfeasible. The classical definition of a wave boundary is the time instant at which the wave crosses a certain amplitude threshold level [2]. The question is respect to which baseline. Where is the null potential? In case of an atrial and ventricular current of injury neither the PQ nor the TP segment represent the null potential, it is most likely that the ST segment is on the null potential. The baseline wander, atrial fibrillation/flutter or high heart rate also cause problem to this definition. Since we are interested in the spread of the cardiac impulse, in the change of potential different between heart muscles, we can use derivatives of the ECG curve to determine the existence of waves and the their boundaries. However there are problems in case of merging $\mathrm{P}$ wave into QRS and J-point into ST-T segment without definite slope changes. There are no exact definitions for these terms in the ECG textbooks either.

It is very difficult to find objective evaluation methods without commonly accepted strict definitions for the ECG wave boundaries. For this reason, the CSE Working Party and the EN 60601-2-51 standard recommend using reference databases and test signals [3-7].

\section{Methods}

Three databases were used for evaluating the threeand twelve-channel ECG measurement programs. The three-channel CSE library consists of real curves with independent QRS onsets and offsets obtained by the referees for all leads. The multi-lead CSE library also includes real curves, but has only one common multichannel onset and offset per record for all the leads. The third set contains the artificial analytical signals of the standard. These databases were used not only for automatic assessment but all records and channels were checked individually and deviations from the references were analyzed.

\subsection{The three-channel CSE database}

Unfortunately, even the references of three-channel database cannot be applied without limitations. Since there is no commonly accepted strict medical and mathematical definition for the wave onset and offset, the referees put the reference points on the base of their own visual judgment. There are huge differences in their decisions. It can be seen in Figure 1 that for $20 \%$ (155 out of 755) of the measurements the maximal differences were more than 6 and $12 \mathrm{~ms}$ for QRS onset and offset respectively. The deviation between referee decisions has 
reached 30 and $50 \mathrm{~ms}$ or more in $10-10$ cases. Two primers are shown in Figure 2 and 3.

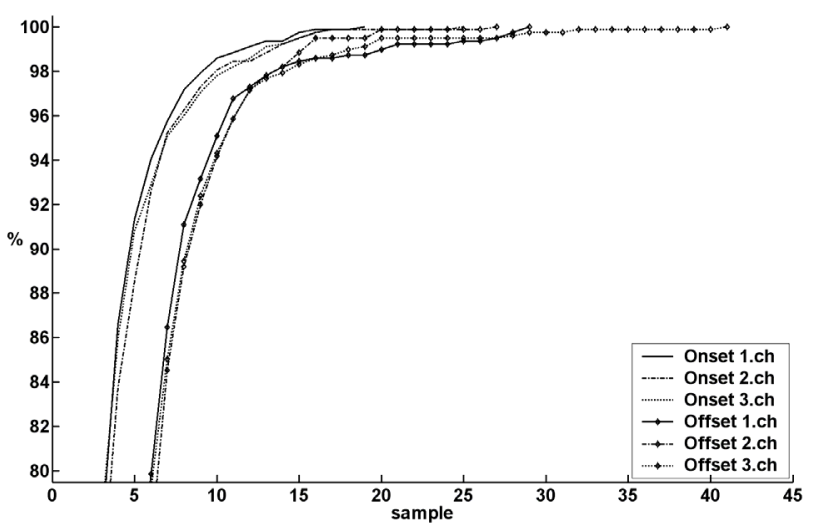

Figure 1. Cumulative distribution function of maximum differences between referees decisions for QRS boundaries

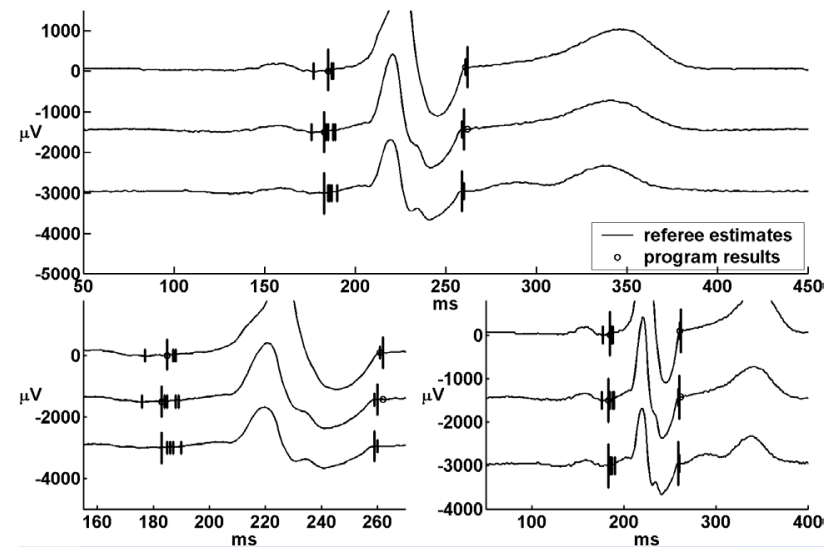

Figure 2. Leads V4 - V6 of the record ea1_044

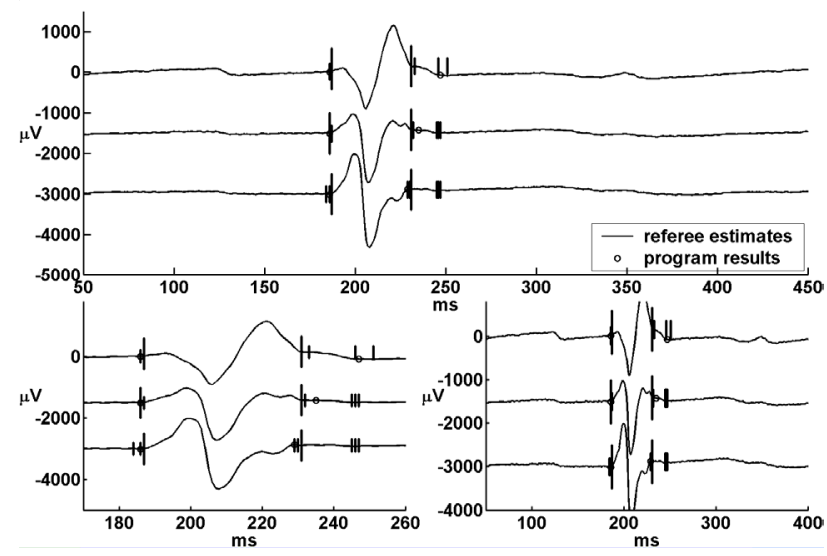

Figure 3. Leads V1 - V3 of the record ea1_016
Furthermore, non-standard gain $(100 \mathrm{~mm} / \mathrm{mV})$ and time $(500 \mathrm{~mm} / \mathrm{sec})$ scales had to be used. For more precise determination of wave boundaries the referees had to use enlarging records, which is uncommon in everyday practice. As shown in Figure 4, the same reference points seem to be simultaneously correct or incorrect at different scales at the same time.
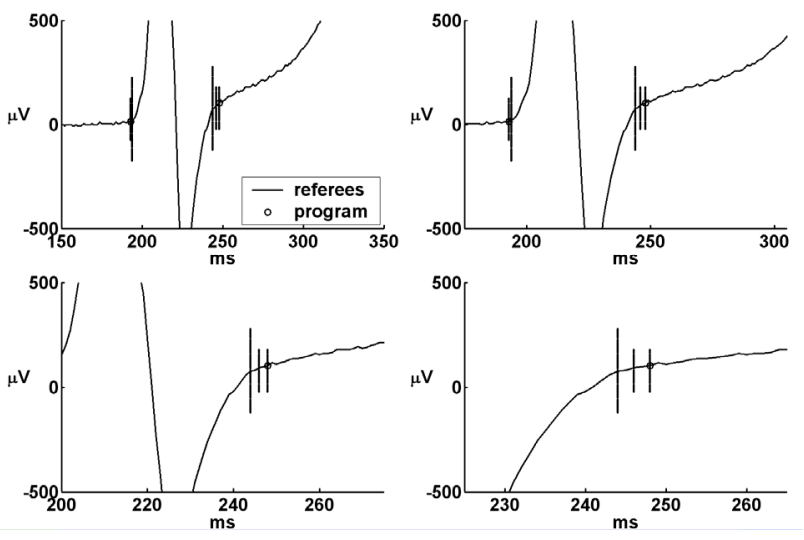

Figure 4. Lead V3 of the record ea1_099

There is inconsistency between acceptance of minimum waves in standard and referee references in the three-channel CSE database. The referees have detected a tiny positive wave at QRS beginning on the record shown in Figure 5, which does not meet the standard's acceptance criteria.

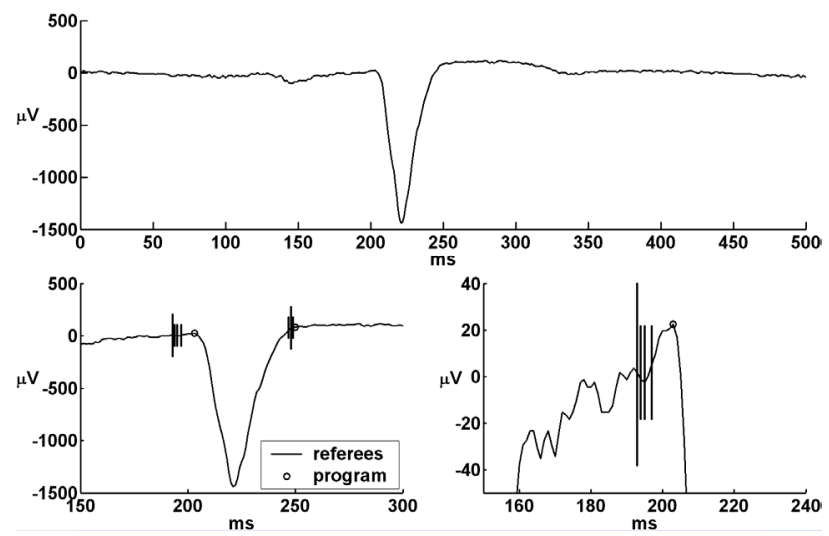

Figure 5. Lead V1 of the record ea1_015

Checking of the reference onsets and offsets of the three-channel library did not reveal the strict mathematical rules that were used by referees reviewing the curves. Fiducial points determined by referees are 
quite often not special points in mathematical term (extreme, inflexion points) and they put them on very unexpected places. For example there are many cases when the referees put the offset of QRS-complex on the middle of an oblique straight line or put the QRS onset on the decline of the Q-wave. Moreover there are several arguable and inconsistent decisions (Figure 6.).

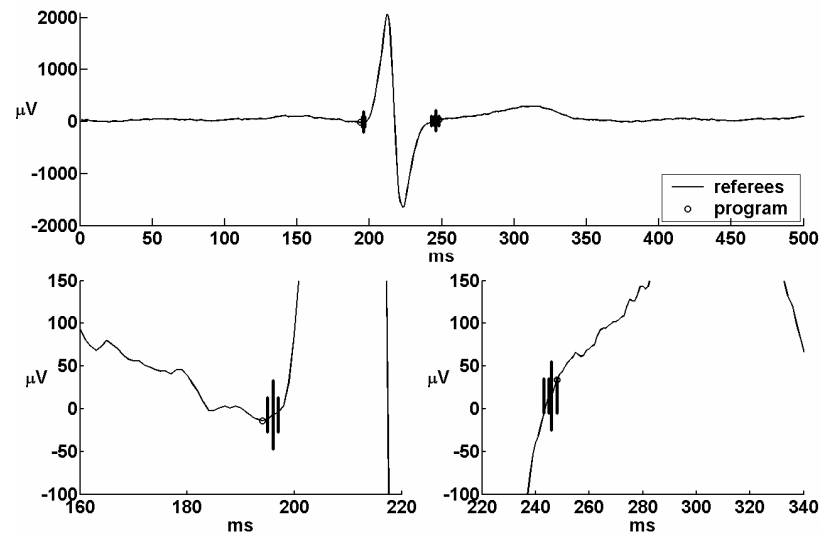

Figure 6. Lead V3 of the record ea1_074

\subsection{The multi-lead CSE database}

The reference values in the multi-lead CSE database are mainly the program medians. Referees reviewed only every fifth record and several fiducial points. This database can be used only for obtaining general view about the multi-channel performance of the analysis programs because sometimes the reference values are subject to doubt.

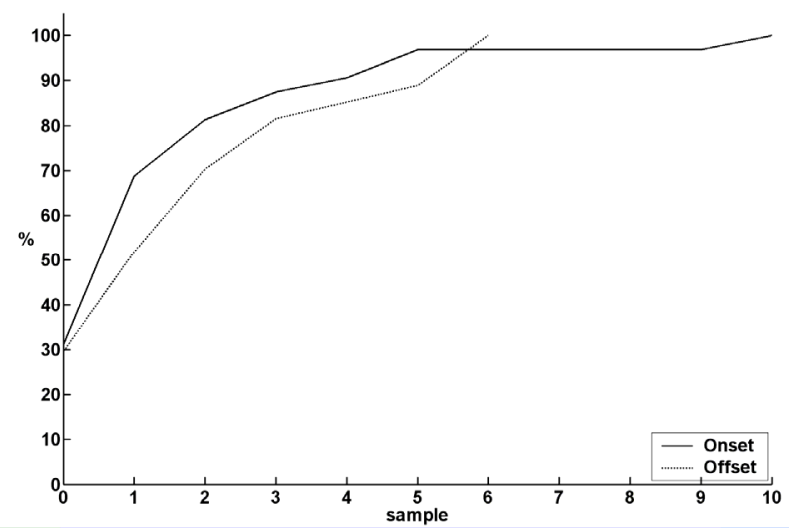

Figure 7. Cumulative distribution function of differences between referee and program medians for QRS boundaries
For example there are differences between the referee and program medians in $65 \%$ and these differences exceed $6 \mathrm{~ms}$ in the 19 and $30 \%$ for QRS onset and offset respectively (Figure 7). In Figure 8 it can be seen the V6 lead of the ma1_105 record. The program QRS onset differs $20 \mathrm{~ms}$ from referee value and obviously wrong. Our analysis program would make a mistake if it puts the QRS onset on the same place.

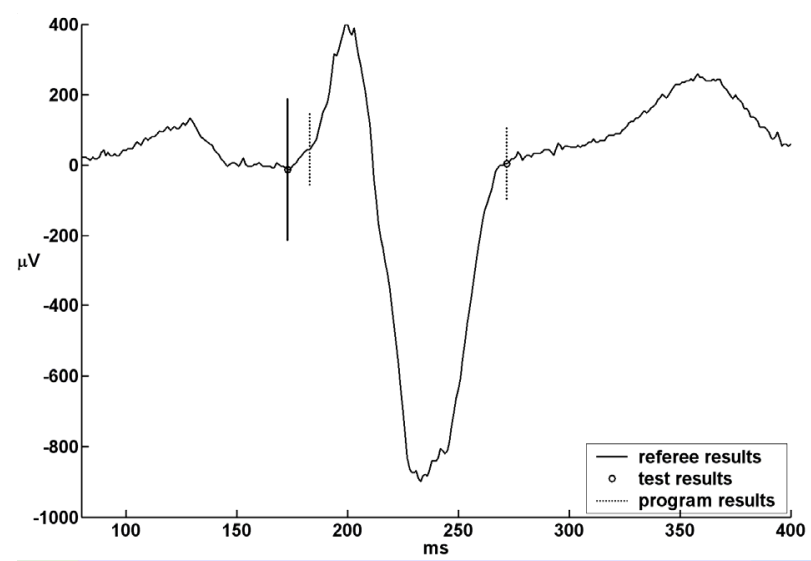

Figure 8. Lead V6 of the record ma1_105

\subsection{Analytical signals of EN 60601-2-51}

The reference values can be used with care since there are some mistakes. For example the minimal acceptance values for QRS waves are $30 \mu \mathrm{V}$ and $12 \mathrm{~ms}$. The III lead of ANE20000 record has small negative initial wave, which is smaller $(15 \mu \mathrm{V}, 12 \mathrm{~ms})$ than these values, even though the stated morphology is QRS (Figure 9) instead of correct RS.
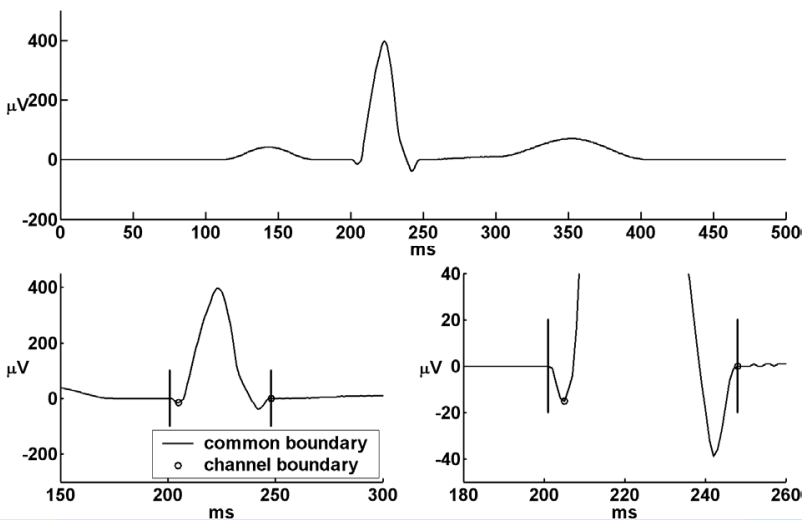

Figure 9. Lead III of the record ANE20000 

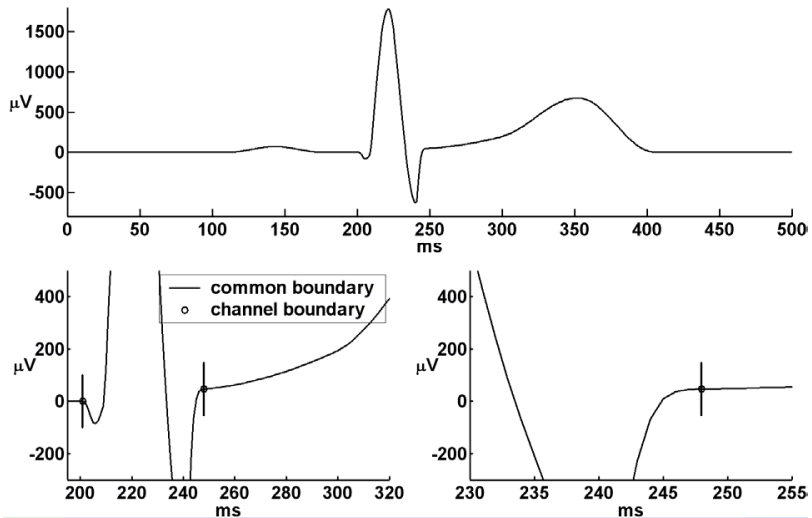

Figure 10. Lead V4 of the record ANE20000

For these signals there are no given the reference points, only reference durations. Our analysis program produces the right QRS duration, but there is doubt about the place of QRS boundaries (Figure 10).

\section{Results}

Strict medical or mathematical definitions of QRS boundaries do not exist. Checking of the reference onsets and offsets of the CSE libraries did not reveal the strict mathematical rules that have been used by referees reviewing the curves. There are surprisingly big differences between referees' decisions. Reference databases and the EN 60601-2-51 standard have several arguable and inconsistent decisions even mistakes.

\section{Discussion and conclusions}

Since there are no strict medical or mathematical definitions of QRS boundaries we would not evaluate and improve performance of ECG analysis programs if there were no reference databases.

The most suitable option for the assessment and development of ECG measurement programs is the threechannel CSE database and it can only be used for assessment of one-channel accuracy. The multi-lead CSE database can be used only for obtaining general view in statistical meaning about the multi-channel performance of the analysis programs and drawing attention to gross detection errors but cannot be used for fine tuning. If we get zero errors during test, it would mean our program does not work correctly.

The CSE databases and the standard analytical ECG curves can be used for automatic evaluation of an ECG measurement program, but the reference data should be used with care. Design engineers have to have appropriate knowledge in both the signal-processing field and electrocardiography.

Obviously the above findings for QRS boundaries detection is mostly true for $\mathrm{P}$ and $\mathrm{T}$ wave boundaries.

\section{References}

[1] The CSE Working party, Recommendations for measurement standards in quantitative electrocardiography. European Heart Journal 1985;6:815825.

[2] Sörnmo L. Laguna P. Electrocardiogram (ECG) processing. Wiley Encyclopedia of Biomedical Engineering. 2006.

[3] Willems JL. CSE atlas, referee results, ACCO Publ. Leuven, 1983.

[4] Willems JL. $9^{\text {th }}$ CSE progress report, ACCO Publ. Leuven, 1989.

[5] Willems JL, Arnaud P, van Bemmel JH, Bourdillon PJ, Brohet C, Dalla Volta S, Damgaard Andersen J, Degani R, Denis B, Demeester M, Dudeck J, Harms FMA, Macfarlane PW, Mazzocca G, Meyer J, Michaelis J, Pardaens J, Poppl S, Reardon BC, Ritsema van Eck HJ, Robles de Medina EO, Rubel P, Talmon JL, Zywietz C. Assessment of the performance of electrocardiographic computer programs with the use of a reference data base. Circulation 1985;71:523-534.

[6] European Standard EN 60601-2-51. 2003.

[7] Deutsche Norm DIN 60601-2-51 (VDE 0750 Teil 2-51). 2004.

[8] Laguna P. Vigo D. Jane R. Caminal P. Automatic waves onset and offset determination in ECG signals: Validation with the CSE database. Computers in Cardiology 1992;167-170.

[9] Laguna P. Vigo D. Jane R. Caminal P. Automatic detection of wave boundaries in multilead ECG signals: Validation with the CSE database. Computers and Biomedical Research 1994;27:45-60.

[10] Kligfield P. Gettes L. Bailey JJ. et al. Recommendations for the standardization and interpretation of the electrocardiogram: part I: the electrocardiogram and its technology: a scientific statement from the American Heart Association Electrocardiography and Arrhythmias Committee, Council on Clinical Cardiology; the American College of Cardiology Foundation; and the Heart Rhythm Society. J Am Coll Cardiol 2007;49:1109-1127

Address for correspondence

Sándor Hargittai

Innomed Medical Inc.

Szabó József utca 12.

H-1146. Budapest

Hungary

E-Mail: hargittai.sandor@innomed.hu 